\title{
Ion Calorimetry: Using Mass Spectrometry to Measure Melting Points
}

\author{
Colleen M. Neal, Anne K. Starace, and Martin F. Jarrold \\ Chemistry Department, Indiana University, Bloomington, Indiana, USA
}

Calorimetry measurements have been used to probe the melting of aluminum cluster cations with 63 to 83 atoms. Heat capacities were determined as a function of temperature (from 150 to $1050 \mathrm{~K}$ ) for size-selected cluster ions using an approach based on multicollision-induced dissociation. The experimental method is described in detail and the assumptions are critically evaluated. Most of the aluminum clusters in the size range examined here show a distinct peak in their heat capacities that is attributed to a melting transition (the peak is due to the latent heat). The melting temperatures are below the bulk melting point and show enormous fluctuations as a function of cluster size. Some clusters (for example, $n=64,68$, and 69) do not show peaks in their heat capacities. This behavior is probably due to the clusters having a disordered solid-like phase, so that melting occurs without a latent heat. (J Am Soc Mass Spectrom 2007, 18, 74-81) (C 2007 American Society for Mass Spectrometry

$\mathrm{A}$ variety of techniques have been developed to examine the physical properties of ions in the gas phase [1]. Interest has focused mainly on properties such as the ground state structure, chemical reactivity, and dissociation energies. There has been little interest in thermodynamic properties such as heat capacities. The reason for this is probably two-fold. First, there has not been a way to measure the heat capacities of isolated ions; and second, there is an expectation that the heat capacities will reveal little of interest. The latter is probably true for small ions that retain the same connectivity between the constituent atoms as the temperature is raised to the point where they dissociate. The heat capacities become interesting for larger ions that can undergo structural transitions or phase transitions (like melting and freezing). For example, unsolvated helical peptides with 10-20 residues can undergo a "melting" transition as the temperature is raised $[2,3]$. Heat capacity measurements would reveal the nature of the transition. A first-order transition occurs with a peak in the heat capacity due to the latent heat, while a second-order transition shows a step rather than a peak in the heat capacity.

Metal clusters are expected to show melting transitions. Here the transition is the finite-size analog of a "real" melting transition rather than the order-disorder transition that occurs for helical peptides. The phase of a dissociating cluster (and indeed any ion) has some interesting [4] and perhaps underappreciated consequences. Some clusters, like $\mathrm{C}_{60}$ and $(\mathrm{NaCl})_{13} \mathrm{Na}^{+}$for example, are particularly stable because they adopt special geometries. We refer to these as structural magic

Published online September 28, 2006

Address reprint requests to Dr. M. F. Jarrold, Chemistry Department, Indiana University, 800 E. Kirkwood Ave, Bloomington, IN 47405, USA. E-mail: mfj@indiana.edu numbers to differentiate them from electronic magic numbers where the stability results from electronic shell closings [5]. If the dissociating cluster is liquid, structural magic numbers should not be prominent in the fragmentation pattern (because stability results from a particular geometry, and the liquid does not have an ordered structure). If, on the other hand, structural magic numbers are prominent in the fragmentation pattern, it implies that the fragmenting cluster is solidlike. Dissociation from a solid-like state can be thought of as the finite size analog of sublimation.

The melting points of small particles are depressed. This was first predicted in 1909 [6], and has subsequently been confirmed many times for particles with $10^{3}$ to $10^{6}$ atoms [7-11]. It is only recently that methods have been developed to investigate the melting of particles with less than $10^{3}$ atoms. In this size regime, where properties change rapidly, measurements must be made on sizeselected particles or clusters, which necessitates the use of mass spectrometry based methods. The pioneering work of Haberland, Schmidt and their collaborators stands out here [12-14]. They developed an experimental method based on monitoring the fragmentation pattern resulting from multiphoton induced dissociation of cluster ions to determine their heat capacities. While their approach has only been applied to sodium clusters with predominantly more that 100 atoms, these studies provided the first experimental information on the melting of particles with atomic resolution.

Here we report heat capacity measurements for aluminum clusters with 63 to 83 atoms. This builds on our previous work, where we have reported heat capacities for aluminum clusters with 49 to 63 atoms [15]. We provide a detailed discussion of the experimental method, and a discussion of the assumptions inherent in the approach employed here. 
a)

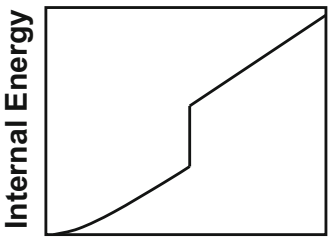

Temperature b)

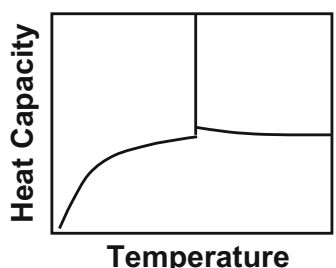

c)

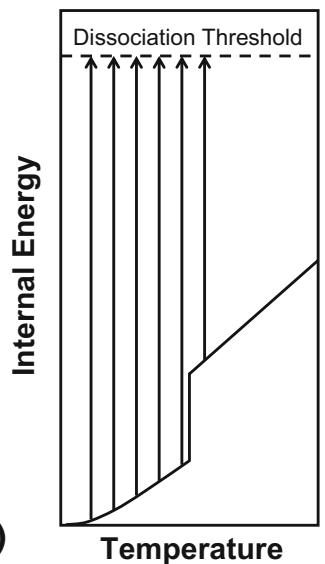

Figure 1. Cartoon illustrating the basic principle behind the measurements reported here. (a) and (b) show the internal energy and heat capacity of a typical crystalline solid as a function of temperature. The step in the internal energy and the spike in the heat capacity are due to melting. (c) shows how measuring the energy required to reach the dissociation threshold as a function of temperature (the arrows) can be used to map out the internal energy and detect the melting transition (the step).

\section{Experimental}

Figure 1 illustrates the basic principle behind the method. Figure $1 \mathrm{a}$ and $1 \mathrm{~b}$ show the internal energy $(\mathrm{E})$ and heat capacity $(\mathrm{C}=\mathrm{dE} / \mathrm{dT})$ of a typical crystalline solid as a function of temperature (T). At low temperature, the sample is solid and the increase in the internal energy with temperature reflects the heat capacity of the solid. At the melting point there is a sudden jump in the internal energy due to the latent heat, which also causes a spike in the heat capacity. Above the melting point the internal energy increases due to the heat capacity of the liquid, which is expected to be slightly larger than for the solid. For a small particle or cluster the melting transition is broadened by finite size effects (the cluster is a small system from a thermodynamic point of view). In our experiments, the melting transition is identified by detecting the jump in the internal energy (or the peak in the heat capacity) due to the latent heat.

How we measure the internal energy is illustrated in Figure 1c. If the internal energy of a cluster is increased it eventually dissociates. We measure the amount of energy required to dissociate the cluster-the vertical arrows in Figure 1c-as a function of temperature. Less energy is required to dissociate the cluster as the temperature is raised because of the increase in the cluster's thermal energy. There is a further sharp drop in the amount of energy required to cause dissociation at the melting transition because of the latent heat (see Figure 1c). The temperature where this sharp drop occurs is used to locate the melting transition. This approach is obviously only possible for clusters that dissociate from a liquid state. If the clusters dissociate before melting (i.e., sublime) we will not be able to detect the melting transition using this method. Tin clusters provide an example of this behavior [16, 17].
Figure 2 shows a schematic of the experimental apparatus. The clusters are generated by laser vaporization of a liquid metal target in a helium buffer gas. The surface regeneration that occurs with a liquid target ensures that the laser always strikes a pristine surface so that the laser or target do not need to be moved to avoid boring a hole in the target. However, the principal advantage of using a liquid metal target is that it provides a signal with much better short term and long-term stability than obtained with a more conventional rod or disk target, which becomes roughened by exposure to the laser. After their formation, the cluster ions are carried through the source region and into the temperature-variable extension by a flow of helium buffer gas. The extension is $10 \mathrm{~cm}$ long and its temperature is regulated by microprocessor based temperature controllers. The controllers regulate liquid nitrogen flow for temperatures below room temperature, and electrical heaters for higher temperatures. The temperature can be adjusted from $77 \mathrm{~K}$ up to around $1200 \mathrm{~K}$. It is critical that the clusters achieve thermal equilibrium with the walls of the extension. A series of tests were performed, where the length of the extension, the size of the exit and entrance apertures, and the buffer gas pressure were adjusted to determine whether thermal equilibrium had been achieved. The tests suggested that the exiting clusters attain the same temperature as the extension walls.

When the clusters are in the temperature variable extension they are in a buffer gas, which provides a means to transfer energy into and out of their internal degrees of freedom. Under these conditions the cluster ions form a canonical ensemble. Because the buffer gas pressure is relatively low (around 10 torr) expansion cooling of the cluster ions as they exit the extension is not important. This was confirmed by doing measurements as a function of the buffer gas pressure and the diameter of the exit aperture. Outside the extension, the cluster ions are no longer in the buffer gas and, thus, no longer form a canonical ensemble. However, if their internal energy is not perturbed as they leave the extension, the cluster's internal energy distribution remains canonical. The cluster ions in vacuum are essentially a "frozen canonical ensemble" characterized by

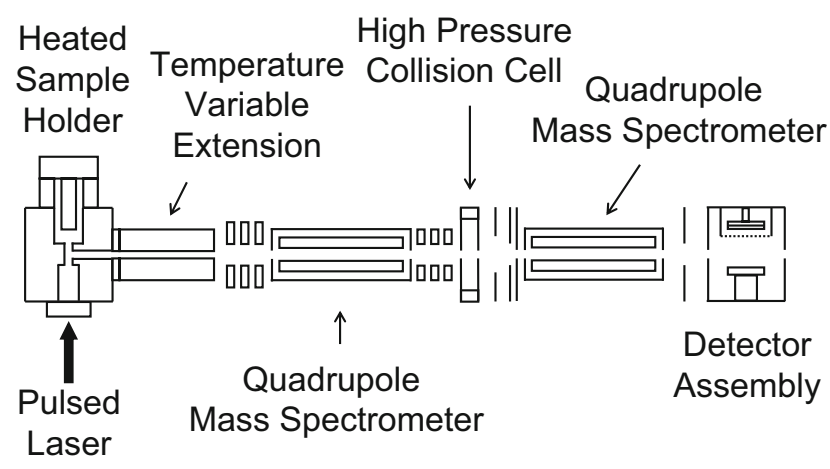

Figure 2. Schematic of the experimental apparatus. 
the temperature of the extension. However, this is only true for a time period that is short compared with the radiative lifetime. Over time, hot or cold clusters will equilibrate to the ambient temperature by black body radiation. While this aspect of our results is not discussed here, cooling of the clusters by black body radiation leads to a signature in the heat capacities that is not discernable for the aluminum clusters studied here, even at the highest temperatures employed. This indicates that radiative cooling is not significant on the timescale of the experiments.

After exiting the temperature variable extension, the cluster ions are focused by an Einzel lens into a quadrupole mass spectrometer (Extrel, Pittsburgh, PA) where a particular cluster size is selected. The sizeselected cluster ions are then focused into a highpressure collision cell containing 1.00 torr of helium collision gas. As the ions enter the collision cell they undergo numerous collisions with the helium, each one converting a small fraction of the cluster ions kinetic energy into internal energy and the relative energy of the collision partner. If the initial translational energy of the cluster ions is high enough when they enter the collision cell some of them are heated to the point where they dissociate. The intact parent ions and the resulting fragment ions are eventually thermalized (stopped) by collisions with the helium, they subsequently cool down to the temperature of the collision gas, and are then drawn across the collision cell by a weak electric field. At the other side of the collision cell, some of the ions exit through a $0.1 \mathrm{~cm}$ diameter aperture, they are subsequently analyzed by a second quadrupole mass spectrometer, and then detected by an off-axis collision dynode and dual microchannel plates.

There are two features of the multicollision-induced dissociation process used to fragment the ions that are critical for the experiments described here. First, multicollision induced dissociation generates a narrow distribution of internal energies [18]. While there is a relatively wide dispersion in the internal energy resulting from a single collision $\left(\Delta \mathrm{E}_{\mathrm{SC}} / \mathrm{E}_{\mathrm{SC}} \sim 1\right)$, the averaging that is inherent in a large number of collisions leads to a distribution that is approximately $N^{1 / 2}$ narrower (where $N$ is the number of collisions). The second critical feature of the multicollision induced dissociation scheme used here is that the fraction of the ion's translational energy that is converted into internal energy is small (a few percent). Helium is chosen as the collision gas for these experiments because it is such a poor collision partner. As a consequence, large changes in the translational energy of the cluster ions are required to compensate for small changes in their average internal energies. This amplification is a critical feature of the experiments.

\section{Analysis of Experimental Results}

Figure 3 illustrates the steps involved in deducing heat capacities from the measurements: First clusters,

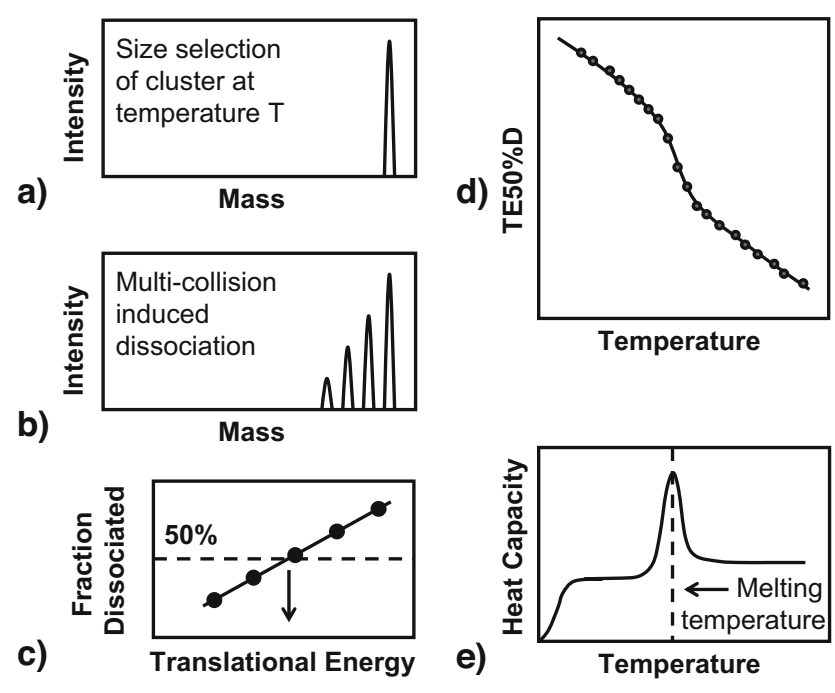

Figure 3. Cartoon showing the steps involved in measuring heat capacities for isolated ions.

at a temperature $\mathrm{T}$, are size selected (a) and dissociated (b). The fraction of the cluster ions that dissociate is determined from the product ion mass spectrum. Measurements are performed as a function of the cluster ions translational energy (c) and a linear regression is used to determine the translational energy required for $50 \%$ of the ions to dissociate $(\mathrm{TE} 50 \% \mathrm{D})$. This quantity, $\mathrm{TE} 50 \% \mathrm{D}$, is then determined as a function of the temperature of the source extension (d). TE50\%D decreases with increasing temperature because, as the temperature is raised, less energy is required to reach the dissociation threshold. The derivative of TE50\%D with respect to temperature is proportional to the heat capacity (to a good approximation). The proportionality constant is the fraction of the cluster ions translational energy that is converted into internal energy. This quantity can be estimated from a simple impulsive collision model, $[19,20]$

$$
\mathrm{F}=\mathrm{C}\left[\frac{n-1}{2 n}\right]\left[1-\left(\frac{\mathrm{m}_{\mathrm{C}}-\mathrm{m}_{\mathrm{B}}}{\mathrm{m}_{\mathrm{C}}+\mathrm{m}_{\mathrm{B}}}\right)\right]
$$

where $n$ is the number of atoms in the cluster, $\mathrm{m}_{\mathrm{C}}$ is the mass of the atoms in the cluster, $\mathrm{m}_{\mathrm{B}}$ is the mass of the collision gas atoms, and $\mathrm{C}$ is an empirical correction factor (obtained from previous cluster dissociation studies [19]). For the aluminum clusters studied here, $\mathrm{F}$ is around $5 \%$. Note that the kinetics of dissociation do not need to be considered because the objective of the experiment is to reproduce the same degree of dissociation (the same internal energy) by balancing the cluster ions initial thermal energy and the energy obtained from collisions.

Figure 4 shows an example of the experimental results. The upper panel in Figure 4 shows a plot of $\mathrm{TE} 50 \% \mathrm{D}$ against temperature for $\mathrm{Al}_{79}^{+}$. Note that be- 

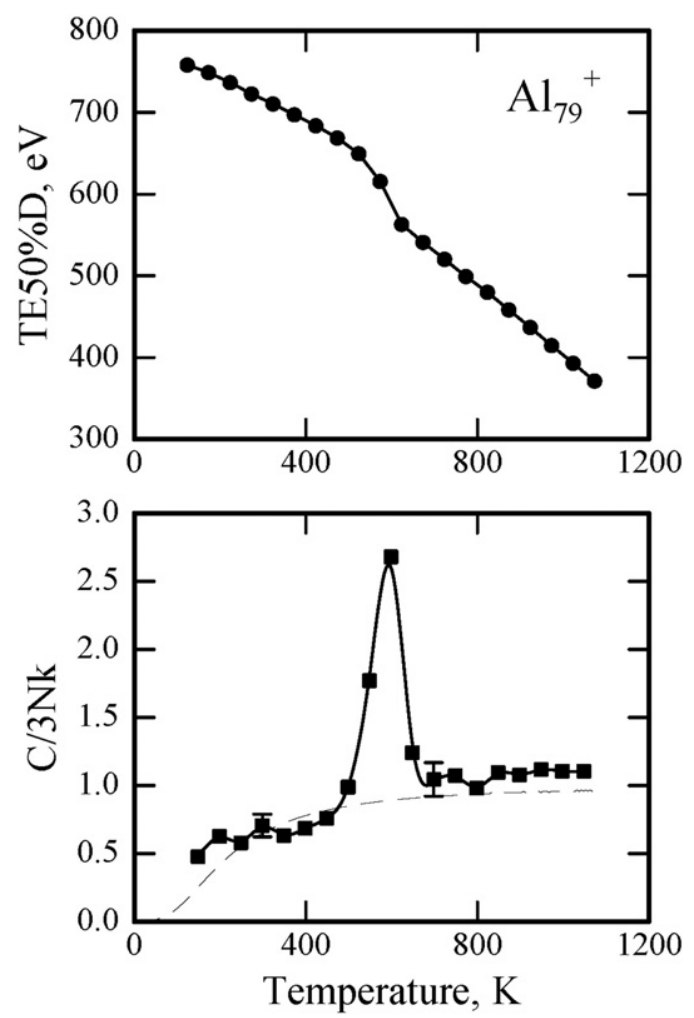

Figure 4. An example of the experimental results. The top panel shows the measured TE50\%D (translational energy for 50\% dissociation) plotted against temperature for $\mathrm{Al}_{79}^{+}$. The lower panel shows the heat capacity determined from the TE50\%D values (see text). The heat capacity is in units of the classical value $3 \mathrm{Nk}$, where $3 \mathrm{~N}=(3 \mathrm{n} \AA 6+3 / 2)$ and $3 \mathrm{n} \AA 6$ is the vibrational contribution, $3 / 2$ is the rotational component, and $\mathrm{k}$ is the Boltzmann constant. The thin dashed line shows the heat capacity calculated using a modified Debye model that incorporates a low-frequency cut-off to account for the finite size of the cluster [21].

cause of the amplification mentioned above, relatively small changes in the thermal internal energy of the cluster ion as a function of temperature lead to large changes in the TE50\%D values. The lower panel shows a plot of the heat capacities derived from the TE50\%D values as described above. The results shown in Figure 4 are an average of three independent measurements. The error bars ( \pm one standard deviation) give an indication of the reproducibility. The heat capacity is given in units of the classical value $3 \mathrm{Nk}$, where $3 \mathrm{~N}=$ $(3 n-6+3 / 2)$ and $3 n-6$ is the vibrational contribution, $3 / 2$ is the rotational component, and $k$ is the Boltzmann constant. The thin dashed line shows the heat capacity calculated using a modified Debye model that incorporates a low-frequency cut-off to account for the finite size of the cluster [21]. The peak in the heat capacity and sharp drop in the TE50\%D values at around $580 \mathrm{~K}$ is the signature of a melting transition. The melting transition is broader than shown in Figure 1 for a bulk system, where the liquid and solid only coexist at a single temperature, the melting point. For $\mathrm{Al}_{79}^{+}$the liquid and solid coexist over a temperature range of around $150 \mathrm{~K}$. This is because the cluster is a small system from a thermodynamic perspective. Away from the melting transition, the measured heat capacities are in reasonably good agreement with the predictions of the Debye model. The heat capacity of the liquid cluster (i.e., temperatures above $\sim 700 \mathrm{~K}$ ) is larger than for the solid cluster (i.e., temperatures below $\sim 450$ $\mathrm{K})$. This behavior is expected as liquids usually have larger heat capacities than solids because of the extra configurational entropy in a liquid.

There are two assumptions inherent in the analysis described above that should be examined further. First, the heat capacity scale is defined using eq 1 . Since this is an approximation, the absolute values of the heat capacities could be subject to error. The high-temperature limit to the Debye heat capacity is the classical value, and the solid clusters are expected to have heat capacities that are close to this quantity. As evident in Figure 4, the measured heat capacities are in reasonably good agreement with the predictions of the Debye model. While this suggests that the method employed here does not lead to a significant systematic error, we should still be cautious about interpreting small differences in the absolute values of the heat capacities. A second assumption that is buried in the analysis described above is that the fraction of the cluster ions translational energy that is converted into internal energy is independent of the structure or phase (solid or liquid) of the cluster and the cross section. The fraction of translational energy converted into internal energy should be independent of the collision cross section because the cross section determines the collision frequency (i.e., the distance traveled between collisions), not the energy transferred in the collisions. The collisions are impulsive (the collision velocities exceed the speed of sound in bulk aluminum) at the relevant collision energies [22]. Thus, the average energy transferred in the collisions should be independent of the structure and phase of the clusters. The heat capacities measured for the liquid clusters are slightly larger than for the solid, but this behavior is usually observed for bulk materials.

\section{Results}

The center of the peak in the heat capacity due to the latent heat is taken to be the melting temperature of the cluster. For $\mathrm{Al}_{79}^{+}$in Figure 4, the melting temperature is around $586 \mathrm{~K}$. This is significantly lower than the bulk melting point of $934 \mathrm{~K}$. Figure 5 shows a plot of the heat capacities measured for $\mathrm{Al}_{54}^{+}$to $\mathrm{Al}_{53}^{+}$as a function of temperature. The results for $\mathrm{A}_{54}^{+}-\mathrm{Al}_{63}^{+}$have been published previously [15]; they are reproduced here so that they can be compared with the results for the larger clusters. Clusters with 54 and 55 atoms have fairly well-defined melting transitions with melting temperatures just above $600 \mathrm{~K}$. For clusters with 56 to 63 atoms, the melting temperature drops and lies between 450 and $550 \mathrm{~K}$. There is also a dip in the heat capacities just before the melting transition 


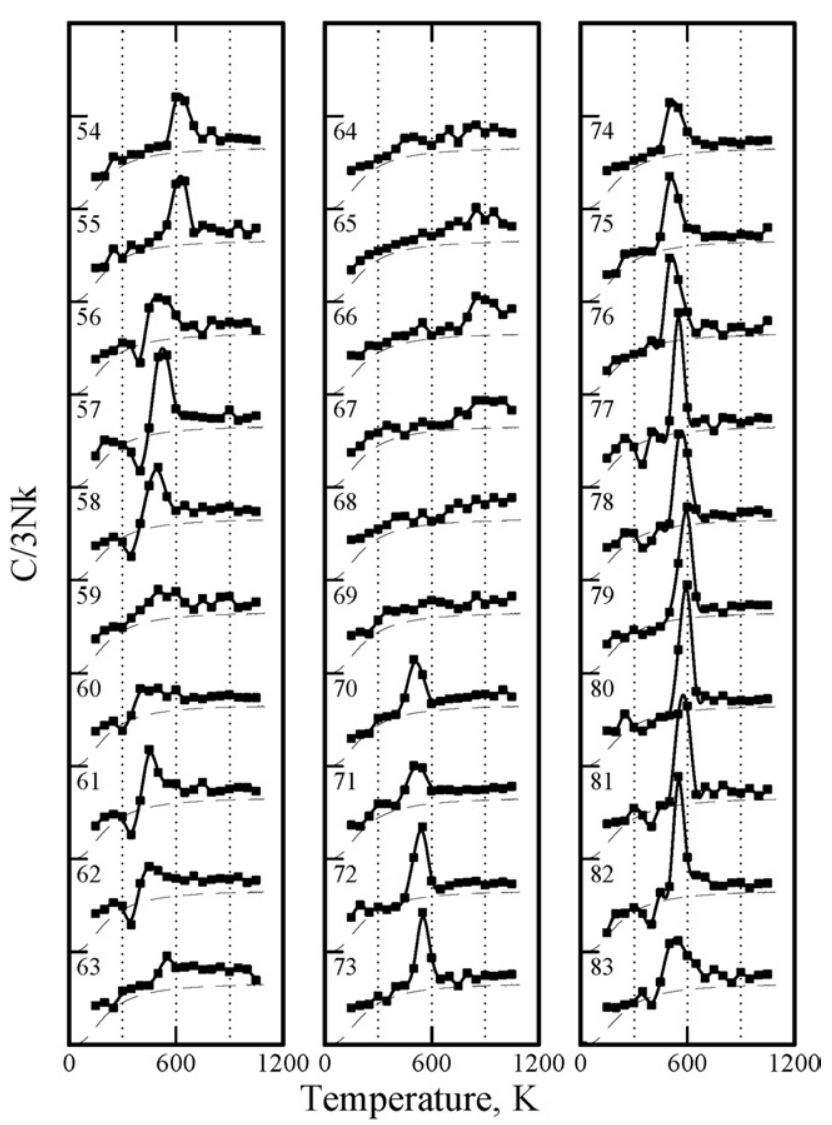

Figure 5. Heat capacity plots for $\mathrm{Al}_{54}^{+}$to $\mathrm{Al}_{83}^{+}$as a function of temperature. The heat capacity is in units of the classical value $3 \mathrm{Nk}$, where $3 \mathrm{~N}=(3 \mathrm{n}-6+3 / 2)$ and $3 \mathrm{n}-6$ is the vibrational contribution, $3 / 2$ is the rotational component, and $\mathrm{k}$ is the Boltzmann constant.

for these clusters. The origin of this dip will be discussed further below.

Clusters with 64 to 69 atoms lack clearly defined melting transitions. For clusters with 64, 68, and 69 atoms the heat capacity plots are almost featureless. For clusters with 65, 66, and 67 atoms there are small maxima at around $900 \mathrm{~K}$. Though small, these maxima are reproducible. If these features are due to melting, there is a spectacular jump in the melting temperature between $62(453 \mathrm{~K})$ and 66 (902 K) atoms. Such a large jump forces us to ask if there could be another explanation for the small maxima in the heat capacities at around $900 \mathrm{~K}$. For example, some gallium clusters have been shown to melt without a significant peak in the heat capacity [23]. Could the cluster melt at a lower temperature and the peaks observed at around $900 \mathrm{~K}$ be due to a liquid-to-liquid phase transition? Liquid-to-liquid phase transitions are very rare in single component liquids. Phosphorus is the only known example, [24, 25] and even there, exceedingly high pressures are required to access the second liquid phase. Thus, apparently the only plausible explanation for the behavior of clusters with 65,66 , and 67 atoms is that the melting temperature suddenly jumps up to around $900 \mathrm{~K}$.
With increasing cluster size, a well-defined melting transition suddenly appears again for $\mathrm{Al}_{70}^{+}$(see Figure 5). The abrupt change on going from $\mathrm{Al}_{69}^{+}$to $\mathrm{Al}_{70}^{+}$is striking. Sharp melting transitions then persist up to $\mathrm{Al}_{82}^{+}$. For $\mathrm{Al}_{83}^{+}$, apparently, the melting transition is starting to broaden out again. Figure 6 shows a plot of the melting temperatures determined from the heat capacity measurements for clusters with 49 to 83 atoms. The dashed line shows the bulk melting point of $934 \mathrm{~K}$. All clusters have melting temperatures that are below the bulk melting point, though for $\mathrm{Al}_{65}^{+}, \mathrm{Al}_{66}^{+}$, and $\mathrm{Al}_{67}^{+}$the melting temperatures are only marginally less than the bulk value (and for $\mathrm{Al}_{64}{ }^{+}, \mathrm{Al}_{68}{ }^{+}$, and $\mathrm{Al}_{69}^{+}$, a melting transition is not detected in the heat capacities).

The area under the peak in the heat capacity is the latent heat (or heat of fusion). For $\mathrm{Al}_{79}^{+}$shown in Figure 4 the latent heat is $291 \mathrm{~kJ} \mathrm{~mol}^{-1}$ or $3.0 \mathrm{eV}$. This is $34 \%$ of the bulk value on a per atom basis. Figure 7 shows a plot of the latent heats determined for aluminum cluster ions with 49 to 83 atoms. The upper plot shows the latent heat per cluster in electron volts. For clusters that show melting transitions, the latent heats vary from around $0.5 \mathrm{eV}$ up to around $3.0 \mathrm{eV}$. The lower plot shows the relative latent heat (the latent heat per atom divided by the latent heat of bulk aluminum). The relative values vary from 10 to $40 \%$ of the bulk value.

\section{Discussion}

While most clusters in the size range examined here exhibit peaks in their heat capacities that can be attributed to melting transitions, for a few (such as $\mathrm{Al}_{69}^{+}$) there is no obvious peak. One possible explanation for this behavior is that the melting transition lies outside the 150 to $1050 \mathrm{~K}$ temperature range examined here. While this could mean that the melting temperature is above the bulk melting point, this behavior is not unprecedented [26]. Another possible explanation is that the clusters melt within the temperature range examined here, but that they melt without a latent heat. This behavior has recently been found in experimental stud-

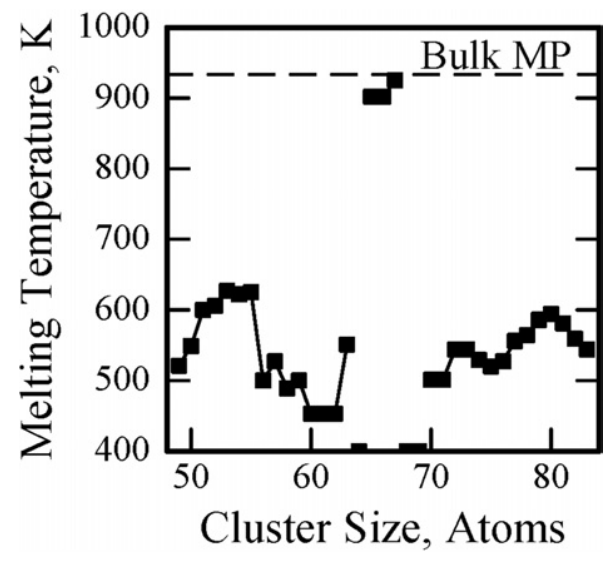

Figure 6. Plot of the melting temperatures (deduced from the center of the peaks in the heat capacity plots) against cluster size. 

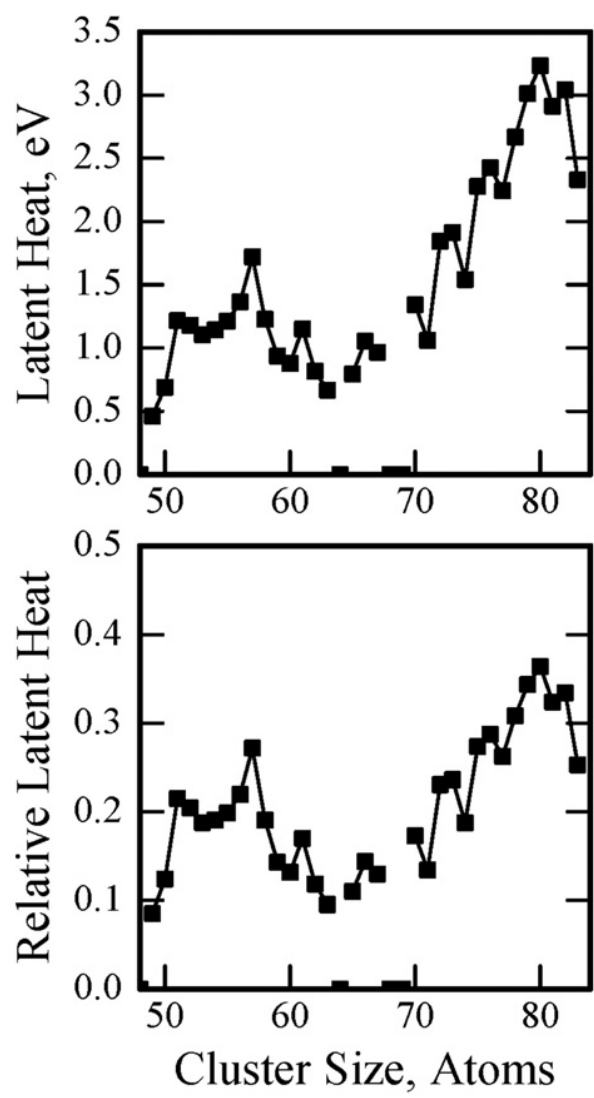

Figure 7. Plot of the latent heats (deduced from the area under the peaks in the heat capacity plots) against cluster size. The upper plot shows the latent heat per cluster in electron volts. The lower plot shows the relative latent heat (the latent heat per atom divided by the latent heat of bulk aluminum, which is $10.71 \mathrm{~kJ}$ $\mathrm{mol}^{-1}$ ).

ies of small gallium clusters [23]. It has also been observed in molecular dynamics simulations where it has been found to occur for clusters that have disordered ground states. Here the "ground state" consists of numerous nearly degenerate structures that lack global symmetry [27-29]. The high symmetry ordered-structures, like icosahedral and fcc, are higher in energy than the lowest energy disordered structures, and so melting proceeds through the finite size analog of a secondorder phase transition (i.e., similar to the melting of a glass). For clusters to melt with a latent heat, (which is analogous to a first-order phase transition in the bulk), there must be a significant gap between the low-energy ordered structures and the disordered ones, as illustrated in Figure 8. For clusters with disordered ground states the question arises whether the ordered structures are destabilized, or the disordered ones stabilized. Because the energy of the disordered structures is not expected to show a strong cluster size dependence, it is presumably the ordered structures that are destabilized in clusters that melt without a latent heat, as illustrated in Figure 8b.

In addition to a peak in the heat capacity due to a melting-like transition, there can be other peaks and dips. Additional peaks can arise from premelting transitions, such as surface premelting, where the surface of the cluster melts before the core. Core premelting, where the core melts before the surface, is also feasible for substances where the liquid is more dense than the solid. Premelting normally results in a small peak that precedes the main melting transition. Premelting is often observed in simulations of cluster melting, [3036] but it appears to be less prevalent in the experimental studies. There appear to be no examples of multiple peaks in the heat capacities in the size range examined here, although some evidence for this behavior was found in studies of smaller aluminum clusters [15].

For most clusters with 56 to 62 atoms, a dip in the heat capacity precedes the peak assigned to the melting transition. A few larger clusters (such as $\mathrm{Al}_{77}{ }^{+}$) also show a dip before the peak (see Figure 5). The dips are attributed to annealing transitions where the clusters convert into lower energy structures as the temperature is raised. Figure 9 illustrates how an annealing transition leads to a dip in the measured heat capacity. Assume that at low temperature the cluster ions are trapped in Structure A, that Structure B is the lowest energy isomer, and that there is a significant activation barrier between $\mathrm{A}$ and $\mathrm{B}$. As the temperature is raised to $\mathrm{T}_{3}$, the internal energy is large enough so that some of A can convert into $\mathrm{B}$ as the clusters travel through the heated extension. When A converts into B, the incipient $B$ contains an internal energy that is larger than possessed by equilibrated $B$ at $T_{3}$. The incipient $B$ will subsequently be thermalized by collisions with the buffer gas. As shown in Figure 9, the amount of energy required to dissociate $B$ at $T_{3}$ is larger than for $A$ at the same temperature. This increase in the amount of energy required for dissociation leads to an inflection in the TE50\%D values, as shown in the plot on the upper right hand side of Figure 9, and a dip in the heat capacity. Because the dip is due to an annealing transition, it is expected to vanish if the clusters are in their lowest energy geometries at all temperatures.

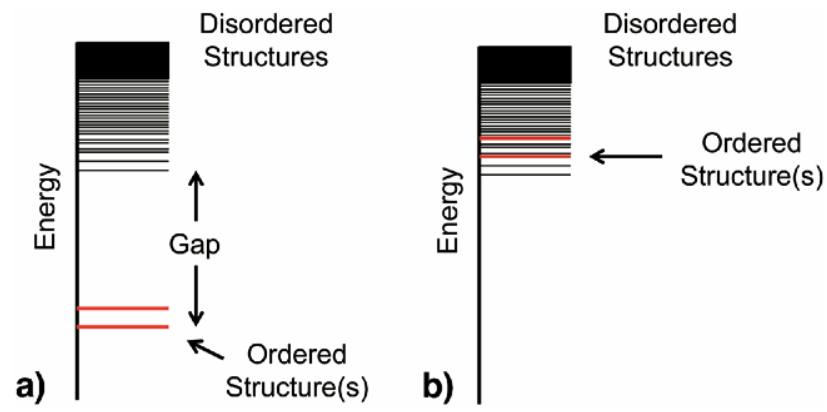

Figure 8. Diagram illustrating the origin of the latent heat in the melting of metal clusters. In (a) there is a significant gap between the lowest energy ordered structures and the disordered ones that evolve into the liquid states. Here a first-order transition occurs with a latent heat. In (b) the ordered structures are embedded in the disordered ones. Here a second-order transition occurs without a latent heat. 

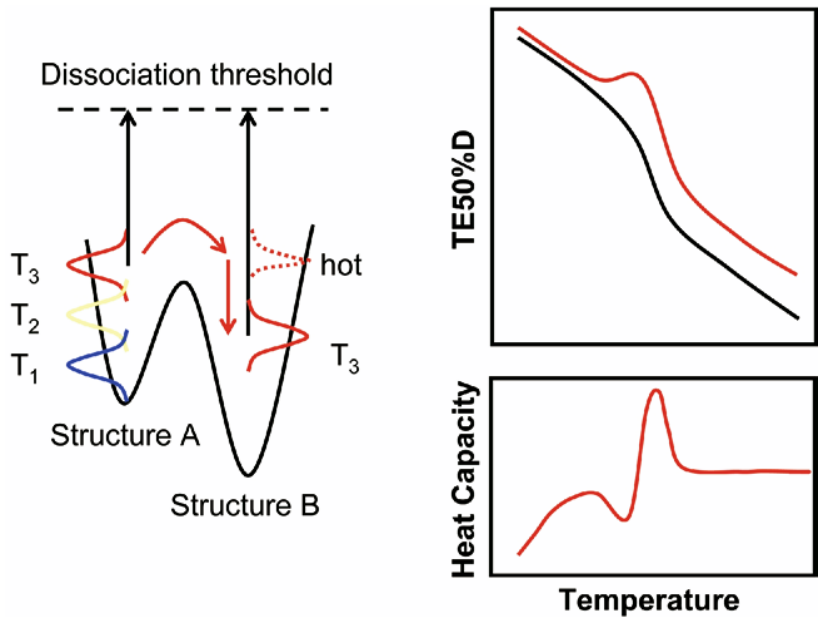

Figure 9. Diagram illustrating how annealing to a lower energy structure (as the temperature is raised) leads to a dip in the heat capacity plot. The diagram on the left shows how annealing into a lower energy structure leads to an increase in the energy required to reach the dissociation threshold. The plot in the upper right shows $\mathrm{TE} 50 \% \mathrm{D}$ values for a system that does not show an annealing transition (black) and one that does (red). An inflection in the TE50\%D values occur at the annealing transition. The lower plot shows the heat capacities derived from the TE50\%D plot that shows the annealing transition.

The difference between the energies of $\mathrm{A}$ and $\mathrm{B}$ (the annealing energy) can be estimated from the area of the dip in the heat capacity plots. Figure 10 shows a plot of the annealing energies obtained for clusters that show significant dips. The annealing energies are all relatively small, less than $1.0 \mathrm{eV}$. Significant dips (annealing transitions) are observed for two groups of clusters, centered roughly around 60 and 80 atoms. These are probably cluster size regimes where the basic geometry of the cluster is changing. If the growing clusters follow a particular growth sequence (i.e., fcc or icosahedral) eventually it may become less favorable or another geometric structure becomes lower in energy. At this point the cluster must change its basic geometry, a process that involves an activation energy. But if enough energy is not provided during cluster growth to accomplish this geometry change, the cluster will become trapped in a metastable geometry, and subsequently anneal when the temperature is raised. Metastable traps are expected to become more prevalent with increasing cluster size.

It is evident from Figure 6 that there are large variations in the melting temperatures with cluster size. The origin of melting temperature variations in small clusters has received considerable attention [33, 37-40], but remains poorly understood. The geometric structure probably plays a role. For example, it is likely that the large drop in the melting temperatures of aluminum clusters with between $n=55$ and 56 results from a change in the geometry at this point. The emergence of the dips in the heat capacities at 56 atoms suggests that a basic change in the geometry occurs at this size. The melting temperatures for aluminum clusters are below the bulk melting point. This is consistent with the prediction of thermodynamic scaling models [6]. But the melting points of clusters in this size regime are not always depressed, both tin and gallium clusters have elevated melting points, $[16,26]$ a result that has been attributed to the clusters having different geometries and different bonding than in the bulk [41-43]. The latent heats for the melting transitions are all smaller than the bulk value. In simulations, the latent heat (per atom) has been found to decrease with cluster size [44], and there is some indication of a systematic decrease in the latent heats in Figure 7. Peaks in the latent heats at 50 to 60 atoms and at around 80 atoms are correlated with local maxima in the melting temperatures. A connection between these quantities is expected. For bulk materials

$$
\mathrm{T}_{\mathrm{m}}=\frac{\Delta \mathrm{H}_{\mathrm{m}}}{\Delta \mathrm{S}_{\mathrm{m}}}
$$

where $\mathrm{T}_{\mathrm{m}}$ is the melting temperature, $\Delta \mathrm{H}_{\mathrm{m}}$ is the latent heat, and $\Delta \mathrm{S}_{\mathrm{m}}$ is the entropy of fusion (the entropy change associated with melting). It is obvious that for a few clusters around $n=66$ (the ones that apparently have high melting temperatures) the correlation between the melting temperature and the latent heat is broken. In this region, the clusters have small or vanishing latent heats so high melting temperatures in this region must be related to a vanishing entropy of fusion.

\section{Conclusions}

The thermodynamic properties of isolated ions have largely been ignored in the past. With the development of ionization techniques that can place large ions in the gas phase, thermodynamic measurements (like heat capacities) have the capability to reveal useful information about structural transitions and phase transitions that can occur in isolated ions. The method employed here to determine heat capacities is based on reproduc-

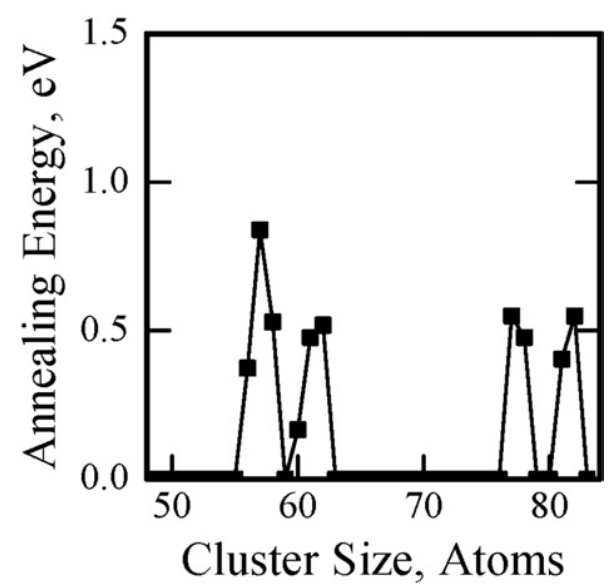

Figure 10. Plot of the annealing energies obtained from the dips in the heat capacities (see text). 
ing the same degree of dissociation (the same internal energy) by balancing the thermal energy (as a function of temperature) with the energy provided by collisions. The multicollision-induced dissociation approach used here leads to a relatively narrow distribution of internal energies (due to averaging). In addition, by using a "poor" collision gas (i.e., one that converts only a small fraction of the ion's kinetic energy into internal energy), large changes in the collision energy are required to compensate for small changes in the ion's internal energy.

Measuring the melting point is straightforward for a bulk material where melting is usually indicated by a visible change in the shape. It is much more difficult to detect melting in microscopic systems. For particles with $10^{6}$ to $10^{3}$ atoms diffraction methods can be employed, and it is well established that the melting point systematically decreases with particle size due to the increase in the surface to volume ratio. Studies in the cluster size regime (less than $10^{3}$ atoms) have been much more challenging, and it is only recently that experimental information has become available. Most of the aluminum clusters in the size range examined here show a distinct peak in their heat capacities, which can be attributed to a melting-like transition. The melting temperatures are all below the bulk melting point and show enormous fluctuations as a function of cluster size. The latent heats (determined from the peaks in the heat capacity) are all less than $40 \%$ of the bulk value. Some clusters (for example, $n=64,68$, and 69) do not show peaks in their heat capacities. This behavior is probably due to the clusters having disordered ground states, so that melting occurs without a latent heat. Dips in the heat capacities, indicating annealing transitions, are observed for narrow ranges of cluster sizes with around 60 and 80 atoms. It is likely that this behavior signals a basic change in the geometry of the cluster.

\section{Acknowledgments}

The authors gratefully acknowledge the support of the National Science Foundation.

\section{References}

1. Encyclopedia of Mass Spectrometry, Volume I: Theory and Ion Chemistry; Armentrout, P. B., Editor; Elsevier Science: Amsterdam, 2003.

2. Okamoto, Y.; Hansmann, U. H. E. Thermodynamics of Helix-Coil Transitions Studied by Multicanonical Algorithms. J. Phys. Chem. 1995, 99, 11276-11287.

3. Kohtani, M.; Jones, T. C.; Sudha, R.; Jarrold, M. F. Proton Transfer Induced Conformational Changes and Melting in Designed Peptides in the Gas Phase. J. Am. Chem. Soc. 2006, in press.

4. Beck, R. D.; St. John, P.; Homer, M. L.; Whetten, R. L. Impact-Induced Cleaving and Melting of Alkali-Halide Nanocrystals. Science 1991, 253, 879-883.

5. De Heer, W. A. The Physics of Simple Metal Clusters-Experimental Aspects and Simple Models. Rev. Mod. Phys. 1993, 65, 611-676.

6. Pawlow, P. Über die Abhängigkeit des Schmelzpunktes von der Oberflächenenergie eines Festen Körpers. Z. Phys. Chem. 1909, 65, 1-35.

7. Peppiatt, S. J. The Melting Point of Small Particles. II. Bismuth. Proc. Roy. Soc. London A 1975, 345, 401-412.

8. Buffat, P.; Borel, J.-P. Size Effect on the Melting Temperature of Gold Clusters. Phys. Rev. A 1976, 13, 2287-2298.

9. Ross, J.; Andres, R. P. Melting Temperature of Small Clusters. Surf. Sci. $1981,106,11-17$.
10. Lai, S. L.; Guo, J. Y.; Petrova, V.; Ramanath, G.; Allen, L. H. SizeDependent Melting Properties of Small Tin Particles: Nanocalorimetric Measurements. Phys. Rev. Lett. 1996, 77, 99-102.

11. Bottani, C. E.; Bassi, A. L.; Tanner, B. K.; Stella, A.; Tognini, P.; Cheyssac, P.; Kofman, R. Melting in Metallic Sn Nanoparticles Studied by Surface Brillouin Scattering and Synchrotron X-Ray Diffraction. Phys. Rev. B 1999, 59, R15601-R15604.

12. Schmidt, M.; Kusche, R.; Kronmüller, W.; von Issendorff, B.; Haberland, H. Experimental Determination of the Melting Point and Heat Capacity for a Free Cluster of 139 Sodium Atoms. Phys. Rev. Lett. 1997, 79, 99-102.

13. Schmidt, M.; Kusche, R.; von Issendorff, B.; Haberland, H. Irregular Variation in the Melting Point of Size-Selected Atomic Clusters. Nature (London) 1998, 393, 238-240.

14. Schmidt, M.; Haberland, H. Phase Transitions in Clusters. C. R. Physique 2002, 3, 327-340.

15. Breaux, G. A.; Neal, C. M.; Cao, B.; Jarrold, M. F. Melting, Premelting, and Structural Transitions in Size-Selected Aluminum Clusters with Around 55 Atoms. Phys. Rev. Lett. 2005, 94, 173401.

16. Shvartsburg, A. A.; Jarrold, M. F. Solid Clusters Above the Bulk Melting Point. Phys. Rev. Lett. 2000, 85, 2530-2532.

17. Breaux, G. A.; Neal, C. M.; Cao, B.; Jarrold, M. F. Tin Clusters That Do Not Melt: Calorimetry Measurements up to 650 K. Phys. Rev. B 2005, 71, 073410.

18. Jarrold, M. F.; Honea, E. C. Annealing of Silicon Clusters. J. Am. Chem. Soc. 1992, 114, 459-464.

19. Jarrold, M. F.; Honea, E. C. Dissociation of Large Silicon Clusters-The Approach to Bulk Behavior. J. Phys. Chem. 1991, 95, 9181-9185.

20. Jarrold, M. F. Drift-Tube Studies of Atomic Clusters. J. Phys. Chem. 1995, 99, 11-21.

21. Bohr, J. Quantum Mode Phonon Forces Between Chain Molecules. Int. J. Quantum Chem. 2001, 84, 249-252.

22. The Relevant Collision Energies are the High Energy Ones, When the Ions First Enter the Collision Cell. The Lower Collision Energies Are Not Impulsive, But They Are Experienced by All Ions.

23. Breaux, G. A.; Cao, B.; Jarrold, M. F. Second Order Phase Transitions in Amorphous Gallium Clusters. J. Phys. Chem. B 2005, 109, 16575-16578.

24. Katayama, Y.; Mizutan, T.; Utsumi, W.; Shimomura, O.; Yamakata, M. Funakoshi, K. A First-Order Liquid-Liquid Phase Transition in Phosphorus. Nature 2000, 403, 170-173.

25. Monaco, G.; Falconi, S.; Crichton, W. A.; Mezouar, M. Nature of the First-Order Phase Transition in Fluid Phosphorus at High Temperature and Pressure. Phys. Rev. Lett. 2003, 90, 255701.

26. Breaux, G. A.; Benirschke, R. C.; Sugai, T.; Kinnear, B. S.; Jarrold, M. F. Hot and Solid Gallium Clusters: Too Small to Melt. Phys. Rev. Lett. 2003, 91, 215508.

27. Sun, D. Y.; Gong, X. G. Structural Properties and Glass Transition in $\mathrm{Al}_{\mathrm{n}}$ Clusters. Phys. Rev. B 1998, 57, 4730-4735.

28. Aguado, A.; Molina, L. M.; Loez, J. M.; Alonso, J. A. Melting Behavior of Large Disordered Sodium Clusters. Eur. Phys. J. D. 2001, 15, 221-227.

29. Westergren, J.; Nordholm, S.; Rosen, A. Melting of Palladium ClustersCanonical and Microcanonical Monte Carlo Simulation. Phys. Chem., Chem. Phys. 2003, 5, 136-150.

30. Nauchitel, V. V.; Pertsin, A. J. A Monte-Carlo Study of the Structure and Thermodynamic Behavior of Small Lennard-Jones Clusters. Mol. Phys. 1980, 40, 1341-1355.

31. Cheng, H. P.; Berry, R. S. Surface Melting of Clusters and Implications for Bulk Matter. Phys. Rev. B 1982, 45, 7969-7980.

32. Aguado, A.; Lopez, J. M.; Alonso, J. A.; Stott, M. J. Orbital-Free Molecular Dynamics Simulations of Melting in $\mathrm{Na}_{8}$ and $\mathrm{Na}_{20}$ : Melting in Steps. J. Chem. Phys. 1999, 111, 6026-6035

33. Calvo, F.; Spiegelmann, F. Mechanisms of Phase Transitions in Sodium Clusters: From Molecular to Bulk Behavior. J. Chem. Phys. 2000, 112, 2888-2908.

34. Lee, Y. J.; Lee, E. K.; Kim, S.; Nieminen, R. M. Effect of Potential Energy Distribution on the Melting of Clusters. Phys. Rev. Lett. 2001, 86, 999-1002.

35. Joshi, K.; Kanhere, D. G.; Blundell, S. A. Thermodynamics of Tin Clusters. Phys. Rev. B 2003, 67, 235413.

36. Calvo, F.; Spiegelman, F. On the Premelting Features in Sodium Clusters. J. Chem. Phys. 2004, 120, 9684-9689.

37. Aguado, A.; Lopez, J. M.; Alonso, J. A.; Stott, M. J. Melting in Large Sodium Clusters: An Orbital-Free Molecular Dynamics Study. J. Phys. Chem. B 2001, 105, 2386-2392.

38. Manninen, K.; Rytkonen, A.; Manninen, M. Influence of Electronic and Geometric Properties on Melting of Sodium Clusters. Eur. Phys. J. D 2004, 29, 39-47.

39. Haberland, H.; Hippler, T.; Donges, J.; Kostko, O.; Schmidt, M.; von Issendorff, B. Melting of Sodium Clusters: Where do the Magic Numbers Come From? Phys. Rev. Lett. 2005, 94, 035701.

40. Chacko, S.; Kanhere, D. G.; Blundell, S. A. First Principles Calculations of Melting Temperatures for Free Na Clusters. Phys. Rev. B 2005, 71, 155407.

41. Joshi, K.; Kanhere, D. G.; Blundell, S. A. Thermodynamics of Tin Clusters. Phys. Rev. B 2003, 67, 235413.

42. Chacko, S.; Joshi, K.; Kanhere, D. G.; Blundell, S. A. Why Do Gallium Clusters Have Higher Melting Points than the Bulk? Phys. Rev. Lett. 2004, 92, 135506.

43. Chuang, F.-C.; Wang, C. Z.; Öğüt, S.; Chelikowsky, J. R.; Ho, K. M. Melting of Small Sn Clusters by Ab Initio Molecular Dynamics Simulations. Phys. Rev. B 2004, 69, 165408.

44. Ercolessi, F.; Andreoni, W.; Tosatti, E. Melting of Gold Particles: Mechanism and Size Effects. Phys. Rev. Letts. 1991, 66, 911-914 